\title{
Multi-scale FE analyses of sheet formability based on SEM-EBSD crystal texture measurement
}

\author{
H. Sakamoto ${ }^{1}$, H. Kuramae ${ }^{2}$, E. Nakamachi ${ }^{3} \&$ H. Morimoto ${ }^{4}$ \\ ${ }^{1}$ Graduate School of Science and Technology, \\ Kumamoto University, Japan \\ ${ }^{2}$ Department of Technology Management, \\ Osaka Institute of Technology, Japan \\ ${ }^{3}$ Graduate School of Life and Medical Science, \\ Doshisha University, Japan \\ ${ }^{4}$ The Furukawa Electric Co. Ltd., Japan
}

\begin{abstract}
The asymmetric rolling is applied to the material process of aluminum alloy sheet to control the micro-crystal structure and the texture in order to improve the mechanical properties. The formability of asymmetrically rolled (ASR) aluminum alloy sheet and conventional symmetrical rolled (SR) one is examined experimentally by employing limiting dome height (LDH) test. The micro crystalline morphological changes, such as the texture evolution under the various proportional straining paths, are observed by using SEM-EBSD measurement. Simultaneously, the failures of the sheets are detected to establish the forming limits in the in-plane principal strains space, such as forming limit diagram. SEM-EBSD measurement results show a significant effect of the strain path on the texture evolutions. It reveals that the ASR sheet evolutes the shear texture more than SR one, and it can be related to improve the formability by employing this asymmetrical rolling. However, the finite element simulations to predict the deformation induced texture evolution of the asymmetrically rolled sheet metals have not been investigated rigorously. In this study, the crystallographic homogenized finite element (FE) codes were developed and applied to simulate the LDH tests, and compared with experimental one. It is shown that this dynamic explicit type crystallographic homogenization FEM code shows a comprehensive tool to predict the plastic induced texture evolution. Keywords: multi-scale, SEM-EBSD, crystal texture, sheet formability, LDH test.
\end{abstract}




\section{Introduction}

The aluminum alloy has superior strength, mechanical properties and corrosion resistance, but it is used less than steel because of the poor formability [1]. Generally, the formability of sheet metal is evaluated by Lankford value (r-value). The r-value is closely related to the crystal texture [2]. The accumulation of $\{111\}$ orientation in plane causes the increasing of $r$-value and the sheet formability are improved $[3,4]$ In the FCC material such as aluminum alloy, $<111>/ / \mathrm{ND}$ are developed by shear deformation. So, the asymmetric rolling process was adopted in order to induce the texture evolution by shear deformation.

In order to clarify the effect of the texture development during plastic deformation on metal forming, crystallographic homogenized finite element (FE) codes [5] were developed. The crystallographic homogenization FE Analysis, however, needs much computation time because of multi-scale and crystalline viscoplastic analysis. So, a parallel analysis method [6] is applied to the crystallographic homogenization FE Analysis and implemented on PC cluster system. The parallel analysis system is implemented with a dynamic workload balancing technique to improve parallel performance. This paper describes application to three-dimensional sheet forming analyses such as LDH test analyses.

\section{Asymmetric rolling process and textures}

The starting material for the ASR tests is hot-rolled plate of A6022 $(6 \mathrm{~mm}$ thickness) alloy. The plate was submitted to asymmetric warm rolling up to the thickness reduction at $250^{\circ} \mathrm{C}$ by two passes, with the a rolling depth from $6.0 \mathrm{~mm}$ to $3.0 \mathrm{~mm}$ in the first pas by thickness reduction of $50 \%$ from $3.0 \mathrm{~mm}$ to $1.0 \mathrm{~mm}$ in the second pass by thickness reduction of $66.7 \%$. The ratio of lower roll velocity to upper roll velocity, the asymmetric ratio $\eta$ was set to 1.75 and 2.0 respectively. The roll diameter in shown in Fig. 1 is $450 \mathrm{~mm}$. The solution treatment is not done to this $1 \mathrm{~mm}$ thickness A6022-ASR plate. The textures of ASR and SR plate were measured by using SEM-EBSD shown in Fig.2. Table 1 shows SEM-EBSD measurement conditions.

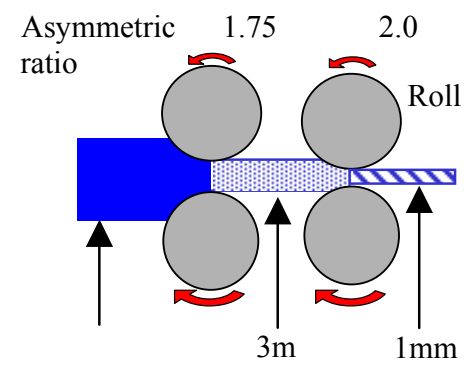

Figure 1: Schematic view of asymmetric rolling. 


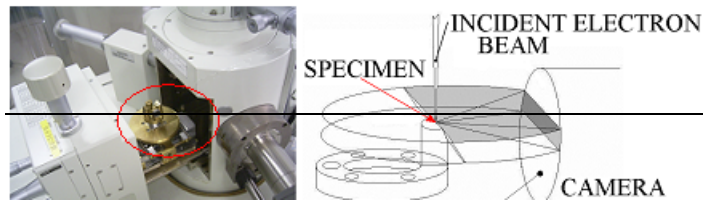

Figure 2: Photograph and schematic view of SEM-EBSD measurement system.

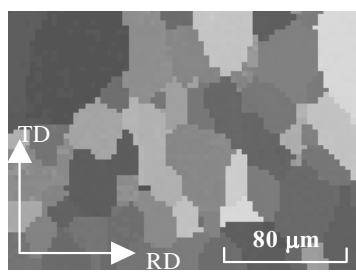

(a)A6022-T43

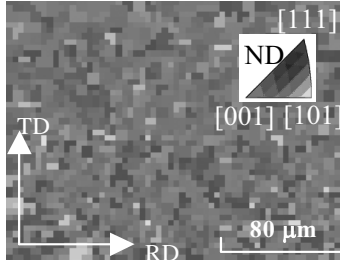

(b)A6022-ASR

Figure 3: Comparison of crystal morphologies between the symmetricallyand asymmetrically-rolled sheet, A6022-T43 and A6022-ASR.

Table 1: $\quad$ SEM-EBSD measurement conditions.

\begin{tabular}{ll}
\hline Measurement magnification & $\times 2000$ \\
\hline Measurement interval (pixel size) & $0.95 \mu \mathrm{m}$ \\
\hline Measured size & $64 \times 50=3200$ pixel \\
\hline
\end{tabular}

Table 2: $\quad$ Grain size and number of grains.

\begin{tabular}{llll}
\hline & \multicolumn{2}{c}{ Grain size $[\mu \mathrm{m}]$} & \multirow{2}{*}{$\begin{array}{c}\text { Number } \\
\text { of grains }\end{array}$} \\
\cline { 2 - 3 } & $\mathrm{RD}$ & $\mathrm{TD}$ & 58 \\
\hline A6022-T43 & 27.8 & 28.9 & 58 \\
\hline A6022-ASR & 6.57 & 6.30 & 1122 \\
\hline
\end{tabular}

Figures 3(a) and (b) show 2D crystal orientation maps of A6022-T43 (symmetric) and A6022-ASR(asymmetric). The average grain size was obtained by intercept method. The each grain size and number of grains are shown in Table 2. From Fig. 3 and Table 2, the reduction of grains size and the strong $\{111\}$ orientations in plane was occurred by asymmetric rolling [7]. 


\section{Formability evaluation of asymmetric rolled sheet on LDH and texture}

Figure 4 shows forming limit diagram (FLD) of A6022-ASR and A6022-T43, where the in-plane maximum and minimum principal strains were plotted in the principal strain space with forming limit curves (FLC), those were obtained by LDH test. This figure shows that the formability of A6022-ASR has been improved especially at the strain path $\varepsilon_{1} / \varepsilon_{2}=\tan 60^{\circ}$ (proportional rolling ratio) and the bi-axial stretching. Fig.5 shows texture evolutions of A6022-ASR under three strain path conditions. The strain paths correspond to the ones in Fig. 4. The (111) orientation is formed as the strain path approaches to the state of Equibiaxial from the one of Plane strain (Fig. 5). The textures in $60^{\circ}$ direction (Fig. 5(b)) and Equi-biaxial (Fig. 5(c)) are obviously different from the texture in Plane strain (Fig. 5(a)) which shows low formability.

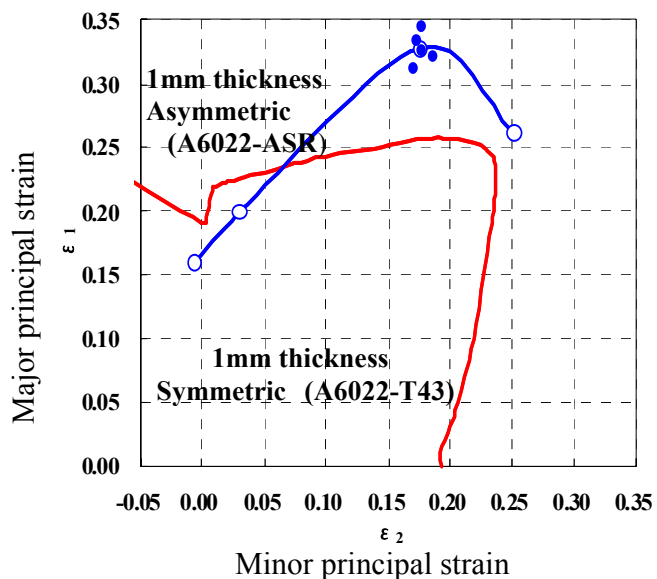

Figure 4: $\quad$ Forming limit Curves of A6022-T43 and A6022-ASR.

\section{Finite element analyses}

\subsection{Elastic/crystalline viscoplastic constitutive equation}

We employed the strain rate dependent crystal plasticity constitutive equation. The crystalline viscoplastic shear strain rate $\dot{\gamma}^{(a)}$ of the power law form defined on the slip system $a$ is expressed as follows:

where

$$
\dot{\gamma}^{(a)}=\dot{\gamma}_{0}^{(a)}\left[\frac{\tau_{\text {eff }}^{(a)}}{g^{(a)}}\right]\left[\left|\frac{\tau_{\text {eff }}^{(a)}}{g^{(a)}}\right|\right]^{\frac{1}{m}-1},
$$

$$
\tau_{\text {eff }}^{(a)}=\tau^{(a)}-\tau_{\text {kin, }}^{(a)}
$$


$\tau^{(a)}, \tau_{\text {eff }}^{(a)}$ and $\tau_{\text {kin }}^{(a)}$ are the resolved shear stress, the effective resolved shear stress and the resolved back stress on the slip system $a$, respectively. $g^{(a)}$ is the reference shear stress, $\dot{\gamma}_{0}^{(a)}$ is the reference shear strain rate, and $m$ is the coefficient of strain rate sensitivity.

The hardening evolution equation of the reference shear stress $g^{(a)}$ is given by

$$
\dot{g}^{(a)}=\sum_{b=1}^{N} h_{a b}\left|\dot{\gamma}^{(b)}\right|,
$$

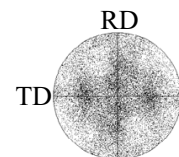

$\{111\}$

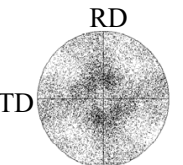

$\{111\}$

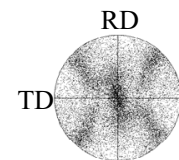

$\{100\}$

(a) Plane strain

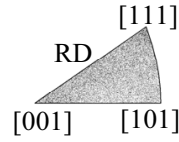

[001] [101]

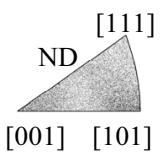

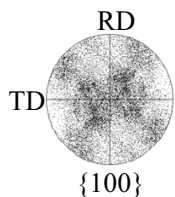
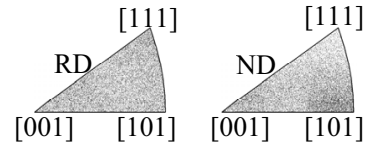

(b) $60^{\circ}$ direction

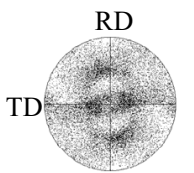

$\{111\}$
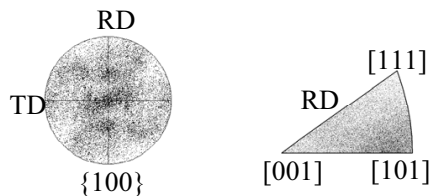

[111]

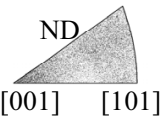

(c) Equi-biaxial

Figure 5: Texture evolutions of asymmetric A6022-ASR $(\mathrm{t}=1 \mathrm{~mm})$ sheet under three strain path conditions.

where $N$ is the total number of slip systems, for the FCC crystal $N=12$, and the BCC crystal $N=48$. $h_{a b}$, the hardening coefficients for hyper tangent saturation equation, are expressed as follows:

$$
\begin{gathered}
h_{a b}=q_{a b} \frac{d \tau(\gamma)}{d \gamma}+\left(1-q_{a b}\right) \frac{d \tau(\gamma)}{d \gamma}(\gamma) \delta_{a b}, \\
\frac{d \tau}{d \gamma}=h(\gamma)=h_{0} \operatorname{sech}^{2} \frac{h_{0} \gamma}{\tau_{s}-\tau_{0}},
\end{gathered}
$$

where the matrix $q_{a b}$ is introduced to describe the self and latent hardenings. The parameters $q_{a b}$ are employed for the coplanar or collinear slip systems as $q_{c}=1$; for the slip systems which have mutually perpendicular Burgers vectors, 
$q_{v}=1.2$, for the others, $q_{l}=1.3$ in the case of FCC aluminum. For BCC steel case, we adopted $q_{c}=q_{v}=q_{l}=1 . \gamma$ is the accumulated shear strain over all the slip systems, $h_{0}$ is the initial hardening modulus, $\tau_{0}$ and $\tau_{s}$ are the critical (initial) and the saturated resolved shear stress, respectively. These values are determined by the parameter identification calculation through the comparison with the experimental results. A three-dimensional polycrystalline macro-continuum is formed by periodic microscopic structures of a representative volume element (RVE) as shown in Fig. 6.

\subsection{SEM-EBSD measured 3D RVE and its micro finite element modeling}

We obtained the distribution of crystal orientation in a 3D parallelepiped box region of the aluminum alloy (A6022) sheet metals, which we call the reference box as shown in Fig. 7. The interval of measurements in three directions, such as RD, TD and ND, was $3.8 \mu \mathrm{m}, 3.8 \mu \mathrm{m}$ and $5.0 \mu \mathrm{m}$, which correspond to the size of the unit voxel. Figure 8 shows initial texture of RVEs for micro finite element modeling.

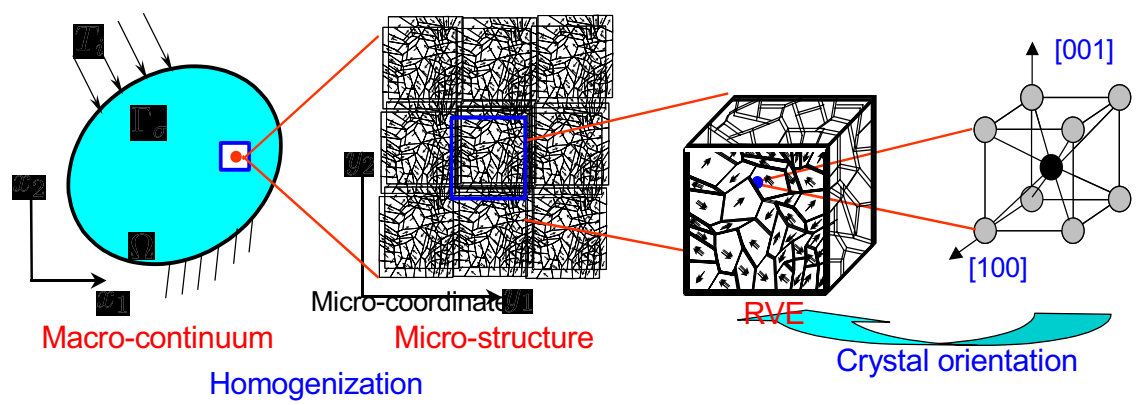

Figure 6: $\quad$ Macroscopic continuum and micro polycrystal structure.

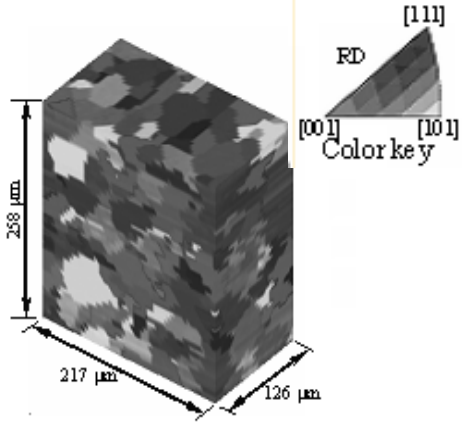

Figure 7: Macroscopic continuum and micro-structure.

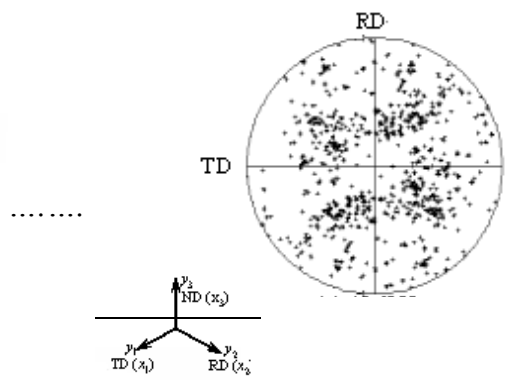

Figure 8: $\quad$ Initial texture of SEMEBSD measured RVEs. 


\subsection{LDH test analyses}

The limiting dome height ( $\mathrm{LDH})$ test is simulated by using the parallel dynamic explicit crystallographic homogenized FE code with SEM-EBSD measured RVE model. The dome is formed by stretching a flat sheet over a flat punch. Tool setup for the problem is shown in Fig. 9. The number of finite elements of macrocontinuum is 448 . FE model of micro-structure with 216 crystal orientations is divided into $27(=3 \times 3 \times 3)$ solid elements. 216 crystal orientations are assigned to each integration point on micro FE model.

In this study, a cluster of AMD Opteron $1.8 \mathrm{GHz}$ PCs connected by Gigabit Ethernet network is used-in the parallel FE analyses. Parallel analysis time and allocated memory on each CPU for the LDH problem are respectively 6.5 hour and $683 \mathrm{MB}$ by using 38 CPUs. Liner speed-up is achieved by the parallel method [6].

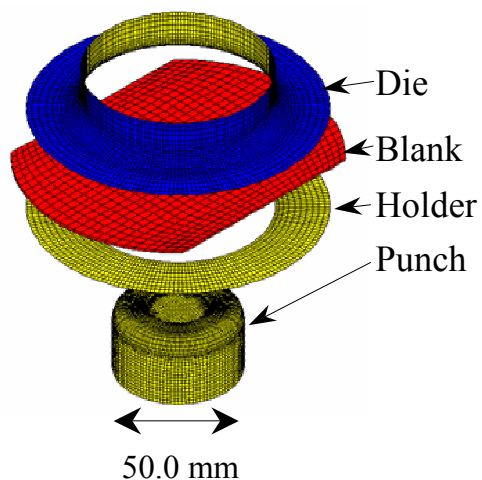

Figure 9: Tool set-up for FE analysis of problem.

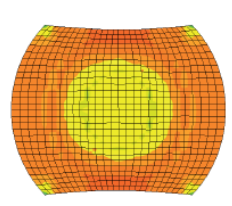

$80 \mathrm{~mm}$ (Plane strain)

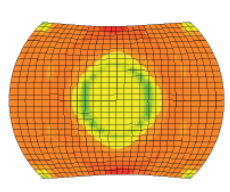

$80 \mathrm{~mm}$ (Plane strain)

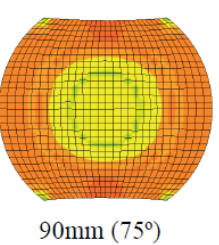

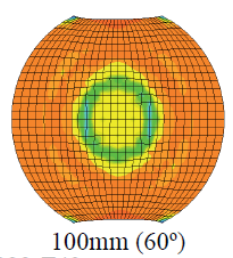

$100 \mathrm{~mm}\left(60^{\circ}\right)$

$110 \mathrm{~mm}$ (Equi-biaxial)
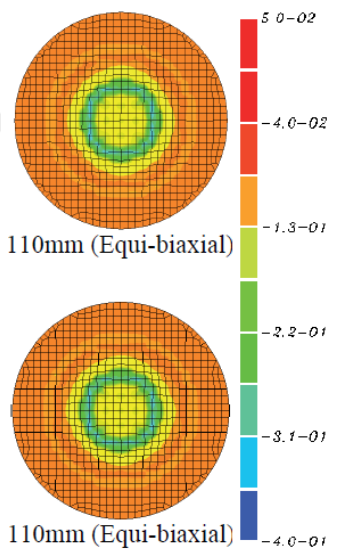

$\mathrm{mm}$ (Equi-biaxial)

(b) A6022-ASR

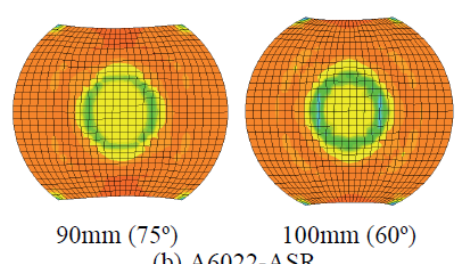

Figure 10: Thickness strain distribution of LDH tests by FEM simulation. 
Figure 10 shows thickness strain distribution at the forming limit. The thickness strain localization occurs around punch shoulder, which is caused by the flat punch. The asymmetrically rolled alloy (A6022-ASR) occurs thinning at plain strain and $75^{\circ}$ strain conditions. The thickness strain distributions of the asymmetrically rolled aluminum sheet metal A6022-ASR are compared with experimental results as shown in Fig. 11. The thickness strain distributions along the centerline of RD direction of blank by FE results are agrees well to the experimental results.

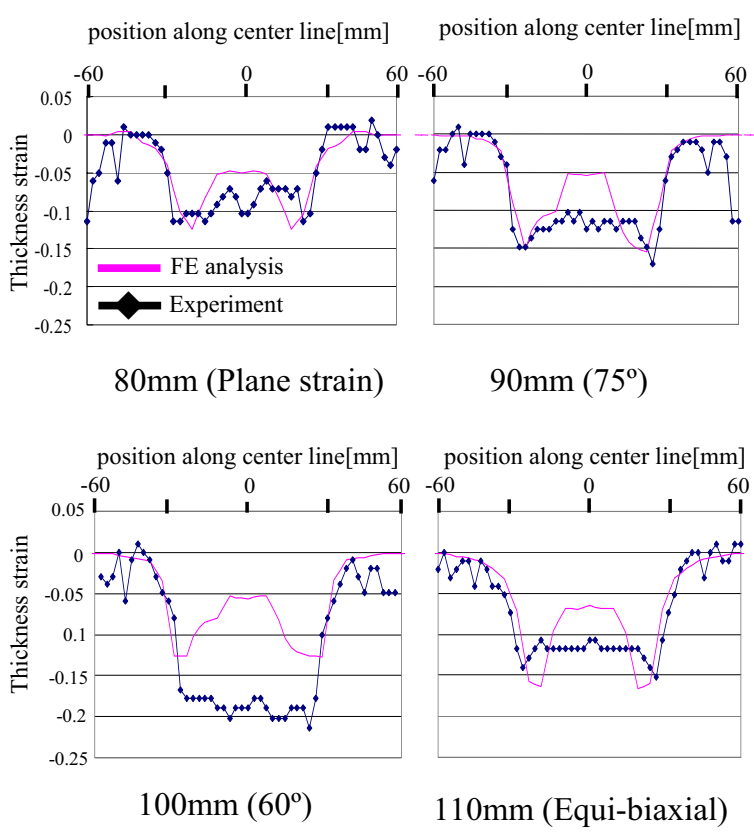

Figure 11: Comparison of thickness strain distribution of A6022-ASR along centre line specimen.

\section{Conclusions}

In this study, a parallel crystalline homogenization algorithm based on the dynamic explicit method has been implemented on PC cluster and applied to LDH test problem for evaluating the automotive sheet metal forming. SEMEBSD measured three-dimensional RVEs are constructed for micro polycrystal model. The forming limit diagram of asymmetrically rolled aluminum alloy A6022-ASR indicates higher formability than the symmetrically rolled aluminum sheet metal A6022-T43 at $60^{\circ}$ and $75^{\circ}$ directions. 


\section{References}

[1] Japan Institute of Light Metals (Eds.), Aluminum Product and the Manufacturing Technology (in Japanese), Japan Institute of Light Metals, Japan, 2001.

[2] Nagashima, S., (Ed.), Texture (in Japanese), Maruzen Co. Ltd., 1984.

[3] Sakai, T., Yoneda, K. and Osugi, S., Microstructure and Texture Control of Al-Mg Alloy Sheets by Differential Speed Rolling, Proc. of the 14th International Conference on Textures of Materials, pp. 597-602, 2005.

[4] Inoue, H. and Inakazu, N., Estimation of r-Value in Aluminum Alloy Sheets by Quantitative Texture Analysis (in Japanese), Journal of Japan Institute of Light Metals, Vol.44, No.2, pp. 97-103, 1994.

[5] Nakamachi, E., Tam, N. N. and Morimoto, H., Int. J. Plasticity, 23, 450489, 2007.

[6] Kuramae, H., Okada, K., Yamamoto, M., Tsuchimura, M., Sakamoto, H. and Nakamachi, E., Parallel Performance Evaluation of Multi-scale Finite Element Analysis based on Crystallographic Homogenization Method, Computational Plasticity part 1, Eds. D. R. J Owen et al., CIMNE, Barcelona, pp. 622-625, 2005.

[7] Nakamachi, E., Development of Multi-Scale Finite Element Analysis Codes for High Formability Sheet Metal Generation, Materials Processing and Design: Modeling, Simulation and Applications (Proc. of Numiform 2007), American Institute of Physics, pp. 215-220, 2007. 